CIENCIA Y SOCIEDAD

Volumen XXVIII, Número 4

Octubre-Diciembre 2003

\title{
APRENDIZAJE Y GEOMETRÍA DINÁMICA EN LA ESCUELA BÁSICA
}

\author{
Dra. Celia Rizo Cabrera* \\ Dr. Luis Campistrous Pérez*
}

\section{RESUMEN}

Se abordan algunas consideraciones sobre la enseñanza de la geometria en la escuela básica, teniendo en cuenta los avances tecnológicos que existen en este momento. El aporte fundamental del trabajo apunta hacia la integración de las calculadoras y las supercalculadoras en el proceso de enseñanza aprendizaje.

\section{PALABRAS CLAVES}

Geometria, tecnologia educativa, educacion.

Investigadores del Instituto Central de Ciencia Pedagogicas del Ministerio de Educación de la República de Cuba.

El presente trabajo se inscribe también en la conjunción de dos líneas de investigación en las que ha incursionado durante más de una década un grupo de investigadores nucleados alrededor de los autores del mismo: la resolución de

Investigadores del Instituto Central de Ciencia Pedagogicas del Ministerio de Educación de la República de Cuba. 
problemas y el uso de la tecnología en la enseñanza de la Matemática.'

El marco teórico de estos trabajos descansa por una parte en la investigación didáctica sobre el desarrollo de la capacidad de resolución de problemas y por otra en la concepción de que las nuevas tecnologías impactan la enseñanza de la Matemática más allá de un simple medio de cálculo. En el caso de este trabajo específico sobre la enseñanza aprendizaje de la geometría en la escuela de educación básica, se incluyen los aportes de los autores en cuanto al trabajo con conceptos $^{2}$, juicios y razonamientos en la escuela y la concepción de un curso de geometría para la escuela de educación bási$\mathrm{ca}^{3}$ en Cuba.

Ambas líneas se desarrollan con un enfoque histórico-cultural de los procesos de enseñanza-aprendizaje, en el que se reconoce el papel de lo social y la interiorización en estos procesos sin desconocer su carácter personal, subjetivo e intransferible.

La aportación fundamental del trabajo apunta hacia la integración de las "calculadoras" y "supercalculadoras" en el proceso de enseñanza aprendizaje, como una herramienta heurística más, que junto a otras estrategias, técnicas y procesos metacognitivos, son utilizadas por los alumnos de manera natural en los procesos de resolución de problemas y adquisición de nuevas estrategias cognitivas. Por supuesto, sobre la base de la necesidad de conducción de estos procesos

1 Ver Tecnología, Resolución de Problemas y Didáctica de la Matemática, ponencia presentada por los autores en el evento Pedagogía 2001 celebrado en Febrero del 2001 en la Ciudad de la Habana.

2 Ver Lógica y Procedimientos Lógicos en la Enseñanza del Dr. Campistrous (Editora interna del ICCP del Ministerio de Educación de la República de Cuba, 1993) y la Tesis Doctoral de la Dra. Celia Rizo, Investigación sobre el curso de geometría basado en las transformaciones y las congruencias, en la Comisión de Grados Científicos del ICCP. 1987).

3 Se está denominado educación básica a la primaria y la secundaria (nueve grados). 
y, por tanto, de su inclusión explícita en el proceso de enseñanza-aprendizaje.

En este material se abordarán, como se plantea en el título, algunas consideraciones que son importantes para esclarecer las posiciones personales de los autores acerca de la enseñanza de la geometría en la escuela básica, teniendo en cuenta los avances tecnológicos que existen en este momento y de los cuales la escuela se puede valer para transformar las formas usuales de organizar y dirigir el proceso de enseñanzaaprendizaje de la matemática, y de la geometría en particular.

Como casi todo el mundo está de acuerdo, desde tiempos inmemoriales hasta la actualidad, la enseñanza y el aprendizaje de la geometría es uno de los aspectos esenciales de la matemática en la escuela de educación general, a lo cual no se puede renunciar. No obstante, es obvio que la forma de enseñar y de aprender geometría tiene que sufrir transformaciones, que van más allá de una simple reorganización del contenido geométrico o de una variante diferente de presentación de los mismos según el punto de vista que se adopte para ello, aunque sea también necesario pensar en ello para poder lograr las transformaciones que se desean lograr.

Ante tal disyuntiva, nuestra opinión, que argumentaremos posteriormente, es que es necesario repensar la estructuración del curso actual de geometría en la escuela de educación general básica, e incluir en la nueva concepción la preparación para el uso de nuevas tecnologías desde edades tempranas, lo cual permitiría concebir el proceso de enseñanza aprendizaje de la geometría en una actividad más productiva y donde el tiempo que se gana puede ser invertido en cosas más útiles encaminadas a la solución de situaciones interesantes y de problemas que favorecerían un mayor desarrollo de sus capacidades intelectuales y de su pensamiento en general. No obs- 
tante, para dar pasos en esa dirección habría primero que buscarle respuesta a una serie de cuestionamientos necesarios que uno se hace sobre cómo dar esos pasos, especialmente en la escuela primaria. Entre ellos:

- ¿Cuáles son los cambios en el sistema de trabajo que habría que producir y en qué edades?

- ¿Cuál sería el objetivo de su introducción en cada caso?

- ¿Sería necesario hacer modificaciones curriculares?

- ¿Qué instrumentación didáctica realizar para que sea exitosa su introducción?

- ¿Qué recursos tecnológicos emplear y cómo hacerlo?

\section{¿Por qué hacer cambios y en qué dirección hacerlos?}

Los cambios que hay que producir, tienen que estar dirigidos a una nueva manera de trabajar estos contenidos donde se puedan explotar más y mejor los recursos tecnológicos actuales y poner a los alumnos en situación activa de aprendizaje y donde se enfrenten continuamente a procesos de búsqueda, planteo de conjeturas, comprobación experimental de ellas, entre otras formas de actuación.

En relación con lo planteado en el párrafo anterior, es necesario discutir cómo se ha estado enseñando la geometría durante miles de años y cómo se puede iniciar un proceso de cambio en ello.

La forma clásica de trabajar la geometría, presenta las figuras estáticas, por tanto aparece siempre una posición particular, una concepción particular, una figura en particular. Esto hace que el alumno forme en su imaginación y siempre presente a las figuras geométricas de una manera concreta. 
En relación con lo anterior, independientemente de todas las cosas que puedan decirse sobre el hecho de que las figuras geométricas son abstractas, todo lo que pueda decirse sobre el hecho de que esas figuras son solamente un caso particular, que no deben asumirse las propiedades de la figura concreta que está viendo, en la práctica el alumno siempre ve una figura y siempre piensa sobre una figura y las propiedades las asocia con una determinada figura.

Por ejemplo, aunque enunciamos que la suma de los ángulos interiores de cualquier triángulo es de 180 grados, siempre el alumno lo va a ver asociado a un determinado triángulo, y difícilmente él va a asumir esa propiedad cualquiera que sea el triángulo, porque va a tener alguna figura concreta en su cabeza.

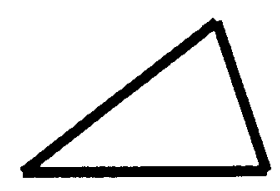

En el trabajo con esta propiedad, que es quizás una de las que más se trabaja, se le hace ver al alumno que da lo mismo que el triángulo sea rectángulo, acutángulo u obtusángulo, pero sin embargo no se tiene tanto cuidado con la figura que sirve de modelo. De igual modo se enuncia, por ejemplo, el hecho de que la distancia entre dos puntos es el menor camino, o que cada lado de un triángulo es menor que la suma de los otros dos, y también eso siempre se ve asociado a una determinada forma de figura.

Ahora bien, cuando las figuras geométricas adquieren la forma de moverse, es decir, adquieren dinamismo, estamos 
en presencia de la geometría dinámica y esta permite que el alumno se forme una idea más general de esas figuras geométricas, que no asocie las propiedades a una forma particular de las figuras.

Por ejemplo, en el caso de la suma de los ángulos interiores de un triángulo, él podrá ver que cuando movemos el triángulo esto hace que se mantenga la suma de sus ángulos interiores y permite, además, precisar el caso especial del triángulo rectángulo y el caso limite que es el caso en que un ángulo se hace 180 grados y los otros dos miden $\mathrm{O}$ grados.

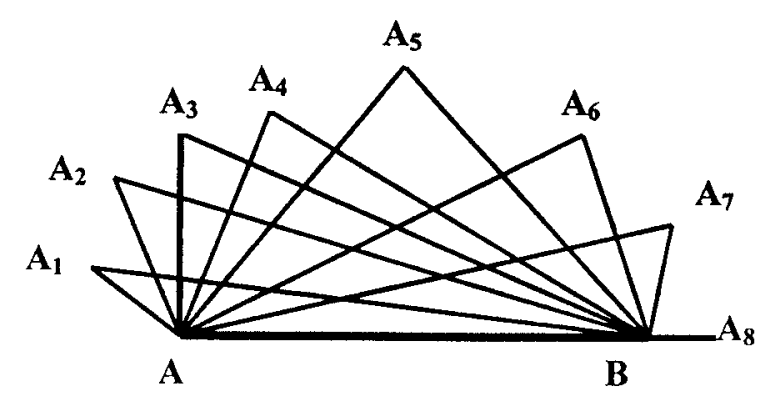

De igual modo sucede con la propiedad de que en todo triángulo, cada lado es menor que la suma de los otros dos, donde con esta variante dinámica se puede, además de comprender de una manera más general la propiedad, también precisar el caso límite, es decir, cuando los tres puntos están en línea recta que es cuando se obtiene la igualdad.

Lo mismo pasa con cualquier tipo de figura, por ejemplo cuando se toma un paralelogramo cualquiera y se analiza la amplitud de sus ángulos opuestos, el alumno podrá apreciar que cualquiera que sea la forma de ese paralelogramo, se va a mantener la propiedad de que esos ángulos son iguales o que tienen la misma amplitud, pero sin embargo, si se toma 
la longitud de los lados consecutivos, los alumnos verán que la propiedad de igualdad de dos lados consecutivos solo se va a cumplir en un tipo muy particular de cuadrilátero (el rombo $y$, en particular, el cuadrado) pues cuando uno lo mueve va a obtener variaciones.

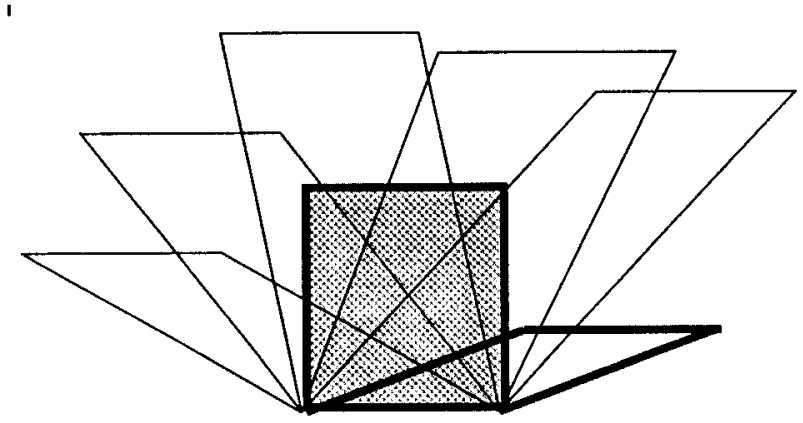

Esto hace que la geometría dinámica permita a los alumnos formarse conceptos mucho más generales acerca de las figuras geométricas y comprender de una manera más completa las propiedades geométricas. De esa manera el alumno no va a asociar ya cada propiedad con una forma particular de la figura.

Otra ventaja de la geometría dinámica es que permite aprovechar plenamente una de las estrategias heurísticas en la solución de problemas geométricos que difícilmente puede ser aprovechada en otros casos, que es la estrategia de "mover la figura". De esta manera el alumno puede mover la figura y conservar ciertas propiedades, y puede formarse una imagen de qué cosa es lo que ocurre al hacer las variaciones y así tener ideas de cómo resolver el problema, es decir, esto permite realizar esta estrategia heurística, ya recomendada por Polya en el año 1944 en la primera edición de su libro How to solve it, que de otra forma es casi imposible de hacer. Lo mismo ocurre con la estrategia heurística de "considerar 
casos particulares", "considerar casos límites", así como "medir y comparar", entre otras, en las cuales al darle movilidad a la figura se hacen visibles de una manera muy natural y se pueden alcanzar esos casos y formarse una idea de cuál puede ser la solución del problema.

Por otra parte, dado que cuando se va a hacer dinámica una figura hay que mantener determinadas condiciones, la geometría dinámica permite fijar las propiedades básicas de las figuras porque para poderla mover y que continúe siendo lo que se quiere que sea, se debe saber exactamente que se puede mover y cómo se puede mover.

\section{¿Qué cambios curriculares habría que hacer?}

$\mathrm{Al}$ igual que se planteó con la calculadora en la escuela primaria ${ }^{4}$ es posible introducir la calculadora y otros avances tecnológicos desde la escuela primaria, y no debe haber limitantes en cuanto a la edad si se precisan bien los propósitos de su uso y esto se hace atendiendo a las características de los escolares según su edad.

La definición de en qué usar esos medios dentro de la geometría en cierto modo se corresponden con algunas de las ya antes definidas para el uso de las calculadoras en la aritmética. Entre ellas pueden citarse:

- Concentrarse en el proceso de resolución de problemas y no solo en el cálculo formal clásico de la geometría, como es el caso de calcular perímetros, áreas, volúmenes, entre otros.

- Explorar, desarrollar y reforzar conceptos y relaciones geométricas.

4 Ver artículo de los autores titulado La Calculadora en la Escueia Primaria, ¿Amiga o Enemiga? 2001. Ponencia presentada en el evento internacional Pedagogía 2001. La Habana, Cuba. 
- Utilizarla como medio heurístico, para la búsqueda de relaciones, planteo de conjeturas de modo de dar acceso a otras formas de pensamiento que van más allá de los algorítmicos propiamente dichos.

- Para objetivar vías de demostración de propiedades de las figuras geométricas.

Para implementar el uso de esta tecnología habría que estructurar un curso de geometría que le dé cabida a lo que hemos denominado geometría dinámica, es decir que dé posibilidades de "darle movilidad a las figuras" además de realizar cálculos si son necesarios.

Para darle respuesta al planteamiento anterior nos remitimos a exponer brevemente tres caminos o vías más utilizadas actualmente para fundamentar un curso de geometría en la escuelas:

- El camino de Hilbert que parte de la congruencia de segmentos y de ángulos.

- El camino de Pieri, que siguiendo a su maestro Peano considera como primario (no definido) el concepto de movimiento.

- El camino seguido por Kagan que partió del concepto de distancia entre dos puntos.

En realidad, estos tres caminos son equivalentes desde el punto de vista de la ciencia matemática, pues a partir de uno

5 De la tesis doctoral de la Dra. Celia Rizo (La Habana, 1987) tomado del artículo "Fundamentación axiomática de la geometría euclidiana" del autor I.M. Yaglom, publicado en 1972 en el libro "Lo nuevo en la matemática escolar", por la Editorial Saber de Moscú (edición en ruso). 
cualquiera de ellos se llega fácilmente a los otros dos, la diferencia estriba en cuál de los tres conceptos: congruencia, movimiento, distancia, se considera el principal o el primario. Lo antes planteado significa que desde el punto de vista matemático, la importancia relativa de uno u otro de estos tres conceptos no tiene ninguna significación, sin embargo, desde el punto de vista didáctico, si tiene una atención preferente.

En el plano didáctico, cuando se va a concretar el contenido de un curso de geometría en la escuela, por razones metodológicas $\mathrm{Y}$ teniendo en cuenta la edad de los niños, nunca la construcción se hace siguiendo en una forma pura ninguno de los tres caminos antes referidos. Por ejemplo, en Cuba, en la actualidad ${ }^{6}$, se parte del camino de Hilbert en que se considera como primario el concepto de congruencia geométrica.

En el camino antes referido para la construcción del curso en Cuba, se hace una simplificación didáctica en el sentido de Euclides en la cual se le da a la congruencia el significado de "igualdad por superposición" o "pueden transportarse uno sobre otro". No obstante, desde los primeros grados se identifica la congruencia de segmentos con la igualdad de longitud (distancia entre puntos) que es el camino de Kagan, y en los grados superiores de la primaria se introducen los movimientos como vía de justificación de la igualdad por superposición, esencialmente cuando se trata de figuras no lineales en donde no se puede identificar o justificar la congruencia con la igualdad de longitud.

Esta vía de construir la geometría en la escuela primaria

6 Ver la tesis doctoral de la Dra. Celia Rizo Cabrera sobre la estructuración del curso de geometría de la escuela general básica en Cuba. Comisión de grados Científicos del Instituto centra de Ciencias Pedagógicas. Ministerio de Educación de la República de Cuba. 1987. 
cubana, desde el punto de vista gnoseológico, comienza en la práctica y culmina en la práctica, en condiciones cualitativamente superiores, después que ha sido enriquecida por un proceso de elaboración intelectual del hombre. Este regreso de nuevo a la práctica constituye, además, el único criterio de verdad. En forma resumida y esquemática este proceso se puede representar así, incluidos algunos procesos del pensamiento que se dan en ese camino dialéctico:

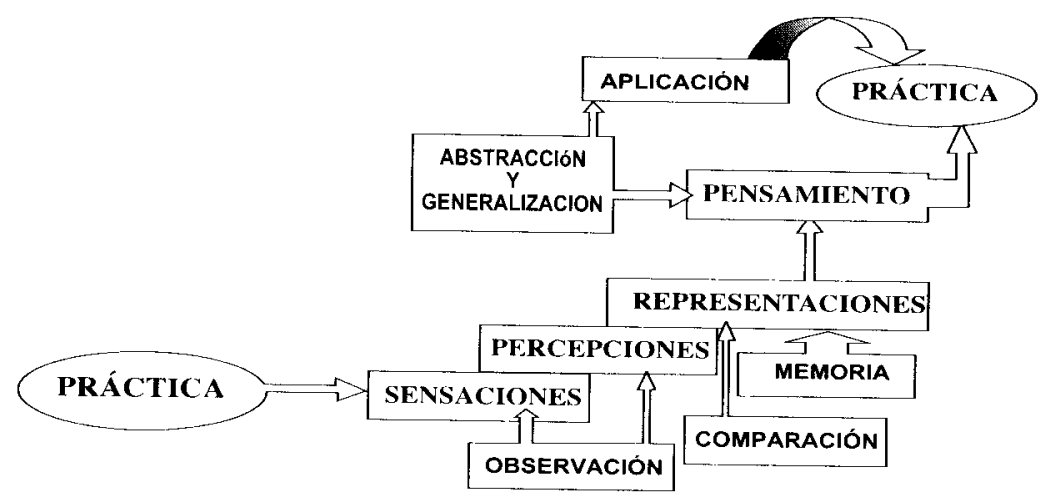

Estas posiciones antes planteadas desde el punto de vista gnoseológico, son también la base metodológica en la concepción de la geometría en la escuela de educación general cubana que se apoyan también en posiciones psicopedagógicas que entre las más significativas se encuentra la concepción acerca del desarrollo y la influencia de lo social en el mismo (Vigotsky).

En el diagrama siguiente se resume esta concepción donde se aprecian claramente dos etapas: Una etapa intuitiva que comprende los cuatro primeros grados de la educación primaria y una etapa racional que comienza en el quinto grado y se 
extiende hasta el último año de la secundaria básica. En ella se hacen visibles las posiciones teóricas antes referidas.

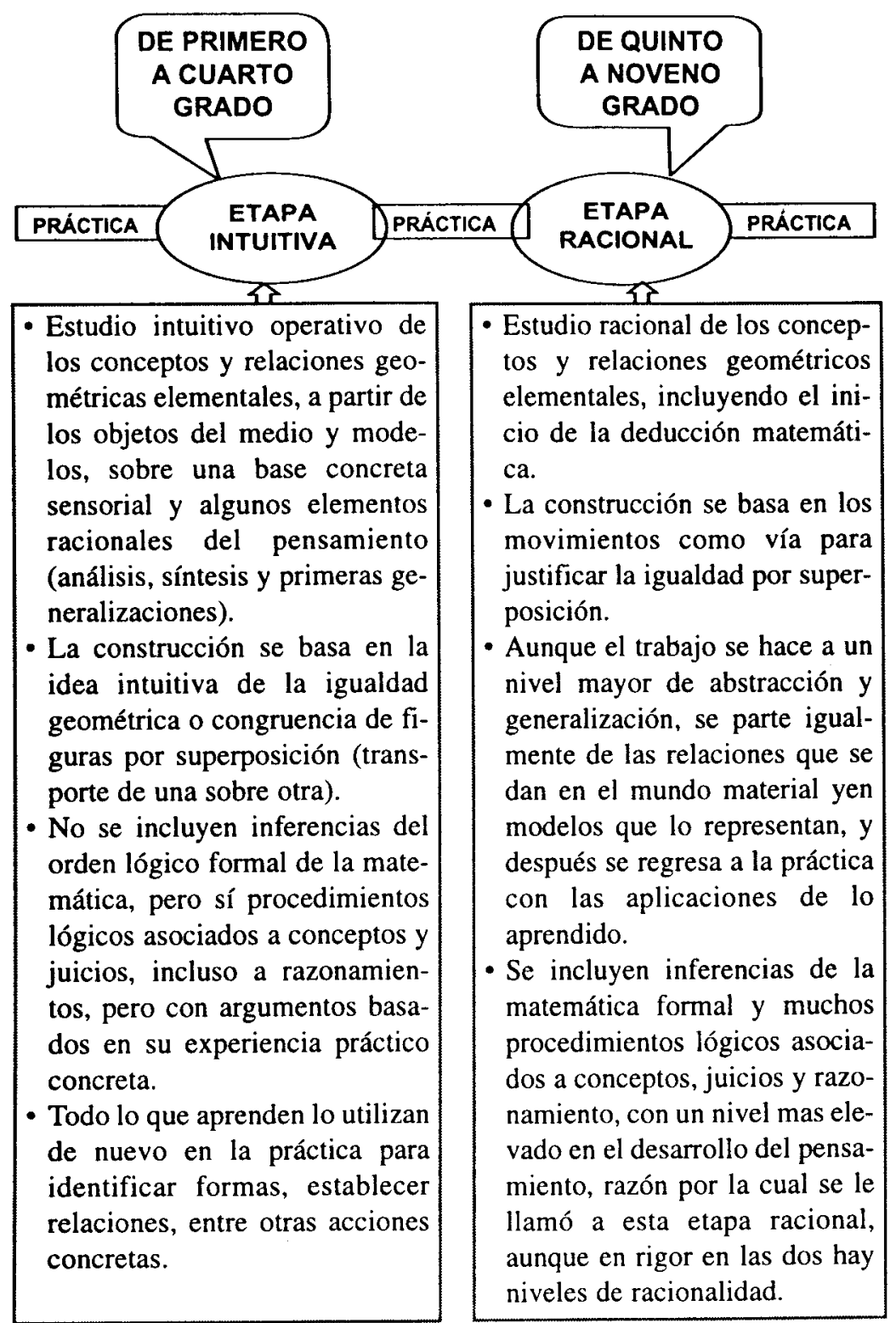


En nuestra opinión, esta concepción permite hacer la introducción de la tecnología, en este caso el empleo de calculadoras, supercalculadoras y computadoras, en el proceso de enseñanza aprendizaje de la geometría, pues con ella existe la posibilidad de "mover las figuras geométricas", es decir, variarlas de modo que adquieran dinamismo, y antes hemos dicho que cuando eso sucede estamos en presencia de la geometría dinámica y ésta permite que el alumno se forme una idea más general de esas figuras geométricas y no asocie las propiedades a una forma particular de las figuras, no obstante habría que incluir algunos elementos de contenido, especialmente en lo que a habilidades se refiere, que permitan también aprender a "mover en una figura o variarla".

Obviamente, para esa introducción, habría que hacer una diferenciación del trabajo entre las dos etapas antes referidas (intuitiva y racional), atendiendo, a su vez a los momentos del desarrollo del escolar atendiendo a sus edades. Los referidos momentos o etapas del desarrollo ${ }^{7}$ en la primaria son los siguientes:

- Preescolar a segundo grado.

- Tercero a cuarto grado.

- Quinto y sexto grado.

En la secundaria, también se puede hablar de etapas del desarrollo del escolar, viéndose el séptimo grado como una transición entre el tercer momento de la primaria y el cuarto momento en la secundaria que ya comprende los últimos grados de la misma.

En la concepción y organización del trabajo pedagógico con

7 Ver el artículo de los autores titulado La calculadora en la escuela primaria, ¿amiga o ene miga? 
estas edades, es muy importante delimitar cada una de estas etapas para poder estructurar y organizar este trabajo de acuerdo al desarrollo a lograr en cuanto a procesos y funciones psíquicas, así como a otros aspectos del desarrollo de la personalidad, que como regularidades de cada momento tienen una diferenciación y por tanto requieren de una atención específica.

Los anteriores criterios que sirven de base para la estructuración del contenido geométrico en la escuela primaria cubana, también serán utilizados para la determinación de las funciones del uso de la tecnología en cada uno de los referidos momentos.

Una posibilidad entonces de estructurar este curso es tener en cuenta las etapas consideradas en la primaria, completarla con la de la secundaria y tener en cuenta, además, las consideraciones hechas con anterioridad sobre las etapas intuitiva y racional en la concepción del curso de geometría en Cuba.

¿Cuáles pueden ser las características del contenido geométrico en cada etapa, con una concepción dinámica, y qué papel podría jugar la tecnología en cada una de ellas?

Cuando hablamos de contenidos geométricos estamos en presencia de un concepto que incluye no solo conocimientos de figuras y cuerpos, sino también de relaciones que se pueden establecer entre ellas y de hábitos y habilidades que permiten operar con esos conocimientos. Dentro de estos hábitos y habilidades se encuentran el de poder realizar cálculos geométricos propiamente dichos, superponer, trazar, medir, comparar, hacer construcciones, entre las más comunes, y otras con un mayor peso intelectual como son las de definir, argumentar, conjeturar, demostrar, entre otras. 
En este trabajo se asumen todas esas habilidades que clásicamente se han incluido en los cursos elementales de geometría, pero se incluyen otras que son necesarias como condiciones previas para poder usar los recursos tecnológicos que estamos proponiendo. Entre ellas se encuentran:

- Construir figuras (rectas, rectas paralelas y perpendiculares, segmentos, ángulos, polígonos, circunferencias) a partir de puntos.

- Determinar puntos libres y variar las figuras a partir de ellos (habilidad rectora).

- Trazar puntos y figuras simétricas.

- Inscribir figuras en otras figuras dadas.

Estas habilidades se pueden ir trabajando desde edades tempranas, usando medios adecuados para ello como los clavijeros o el geoplano (se puede construir fácilmente con un pedazo de madera cuadrada y puntillas equidistantes unas de otras en un arreglo rectangular (cuadrado). Se puede hacer de $9 \times 9$, con un total de 81 puntillas, o de cualquier cantidad impar. Este medio se complementa con ligas o elásticos que son los que le van a dar la movilidad.

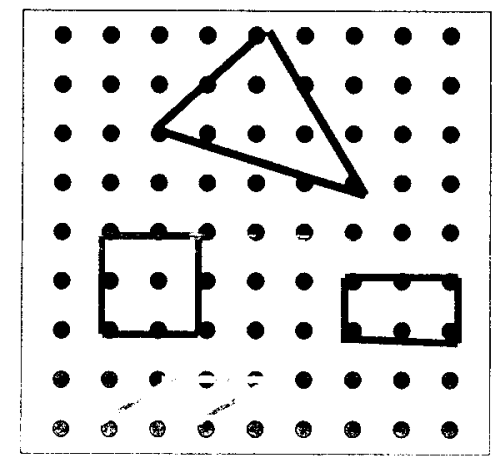


En él los alumnos pueden ir formando figuras con las ligas y las puntillas, y empezar el análisis de propiedades y en cada etapa o momento de desarrollo en la primaria se puede utilizar con variados fines.

A continuación se describen brevemente las funciones de cada etapa del desarrollo, en lo que corresponde a la preparación para el uso de recursos tecnológicos y el uso propiamente dicho de los mismos.

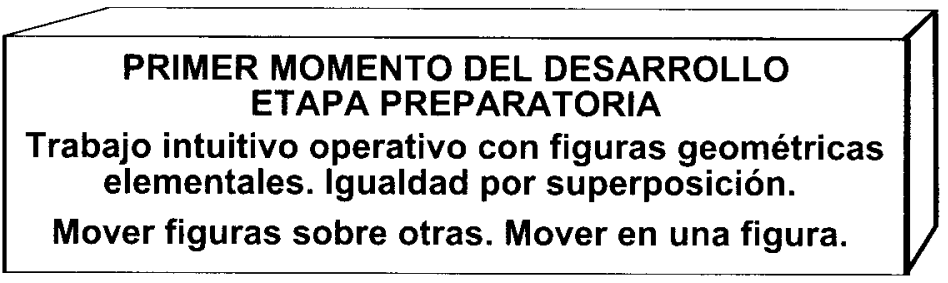

Inicio de las primeras ideas sobre la movilidad de las figuras para comprobar experimentalmente relaciones de congruencia. Uso de clavijeros y ligas para ir desarrollando la habilidad de "mover' en una figura (GEOPLANO). Reproducción en papel cuadriculado. Superponer, medir, comparar.

Por ejemplo, se puede mandar a formar un cuadrado en el geoplano. Después que lo tengan (aquí de entrada se están reforzando las propiedades del cuadrado, al menos la percepción que tienen de esa figura si es que se trata de primer grado donde todavía solo lo identifican por su forma memoria perceptual pero no han analizado propiedades), se les puede indicar que:

- dejando el lado de abajo fijo del cuadrado original, formen un rectángulo (no cuadrado),

- dejando el lado izquierdo fijo, formen un rectángulo diferente al anterior, 
- varíen el cuadrado original y lo conviertan en un triángulo, dejando fijos el lado de abajo y el izquierdo.

Por último se les puede dejar que a partir del cuadrado original formen libremente figuras diferentes y digan de qué figura se trata (si es conocida) y cómo la han obtenido.

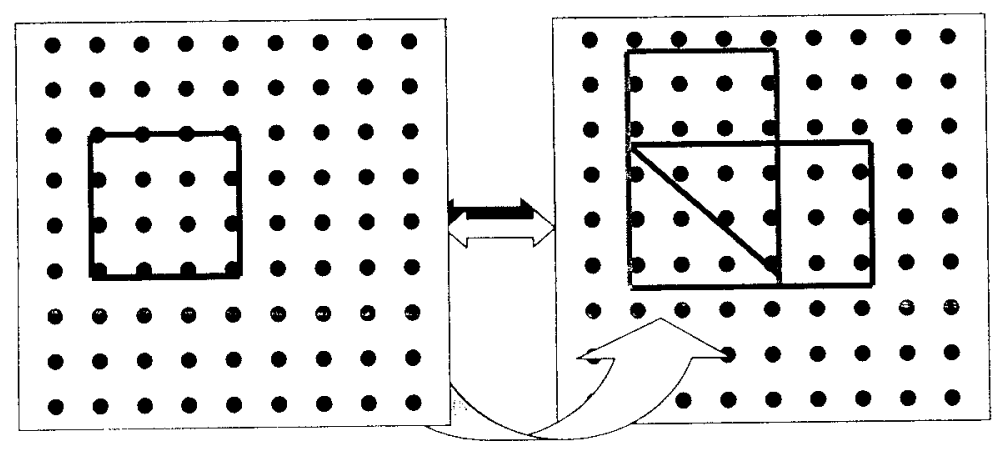

Otra actividad que se puede hacer es dado un triángulo que ellos van a representar en el geoplano, dibujarlo en el papel cuadriculado conservando su forma y tamaño. Después mover el punto de arriba (el vértice superior) de modo de formar muchos triángulos con un mismo lado (el de abajo) e ir reproduciendo lo que hacen en el papel cuadriculado. Esto permite hacer otras actividades como las de medir (o superponer usando una tira de papel como transportador o la misma regla) y comparar los lados de los triángulos que van obteniendo, de modo que puedan concluir cuándo son iguales (congruentes) y cuando no, en cada caso.

Este tipo de actividad permite ir obteniendo casos especiales (rectángulos, isósceles, entre otros), aunque todavía sea prematuro denominarlos de ese modo, pero que se puede lla- 
mar la atención, por ejemplo, que hay casos en que los lados son iguales (isósceles), hay otros donde un lado coincide con un lado de la cuadricula que no es el que está fijo (rectángulo), entre otras cosas, y que si siguen moviendo cada vez más, pueden llegar a "perder el triángulo", o sea obtienen un caso límite de un triángulo degenerado.

El control se puede hacer por parejas o por el mismo equipo (pequeños grupos de alumnos en que se puede descomponer el grupo original) si se organiza la actividad de esta manera, o por el propio maestro en caso de otra forma de organización.

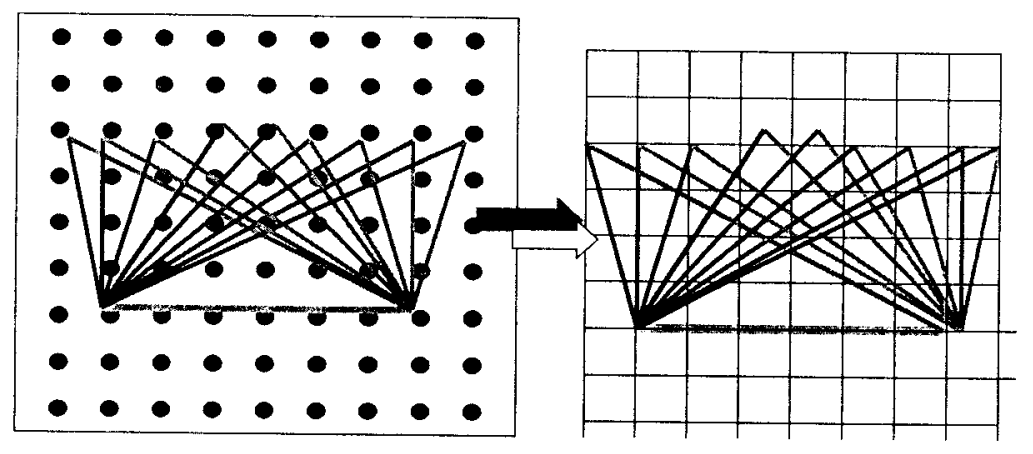

En esta etapa no se propone ningún uso de la tecnología para el caso de la geometría, es solo una etapa de preparación donde lo que se pretende es ir sentando las bases de una geometría dinámica para en un futuro, si poseen los medios, puedan usar la tecnología que estamos proponiendo. En ella se plantea la realización de "simulaciones" con el medio denominado geoplano y donde se puede iniciar el desarrollo de algunas de las habilidades antes planteadas.

En caso de no poseer después estos recursos, siempre se obtiene la ganancia de una mayor flexibilidad del pensamien- 
to de los alumnos y su posibilidad de una mayor comprensión cuando se les plantee que algo se cumple para cualquier tipo de figura y no solo para aquella que han dibujado para representar una situación dada.

No obstante, no se excluye la posibilidad de usar determinados software que se pueda diseñar con carácter instructivo en un entorno lúdico, pero que en un primer momento no sería de utilización masiva.

Una segunda etapa sería la que se corresponde con el segundo momento de desarrollo (tercero y cuarto grados de la primaria con edades entre 8 y 9 años aproximadamente). En esta etapa se propone también el uso del geoplano, además de los medios normalmente utilizados para el trabajo en geometría en estos grados como son regla, cartabón, tijera, entre otros.

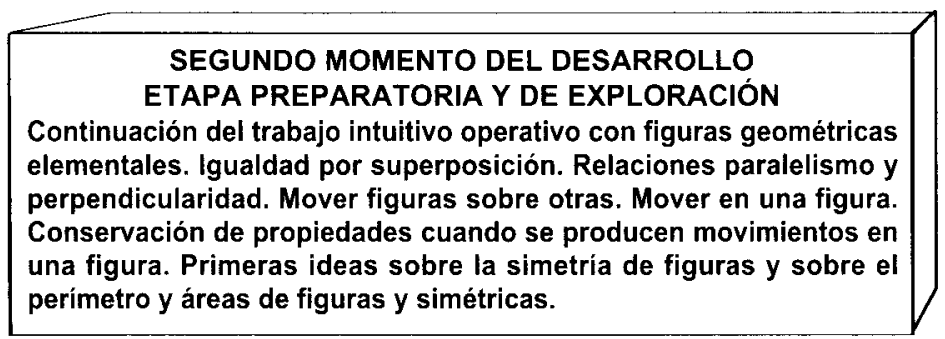

Ampliación de las ideas sobre la movilidad de las figuras para comprobar experimentalmente relaciones de congruencia, y conservación de otras propiedades como el paralelismo y la perpendicularidad. Continuación del uso del geoplano para ir desarrollando la habilidad de "mover" en una figura. Reproducción en papel cuadriculado. Superponer, medir, comparar.

En esta etapa ya se pueden empezar a determinar paralelas 
y perpendiculares que pasen por un punto o que lo sean a un segmento dado. Se puede usar también el geoplano para variar condiciones dadas.

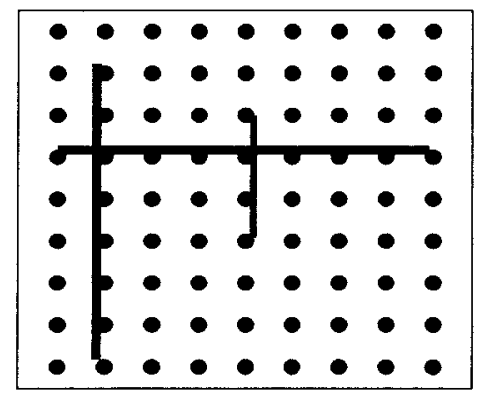

Estas ideas van a permitir ir preparando los conceptos de puntos y figuras simétricas, que son muy importantes desde el punto de vista geométrico, pero que también permiten desarrollar la habilidad de moverse a lo largo de lados y diagonales de cuadraditos (derecha e izquierda y viceversa, arriba y abajo y viceversa), que es muy útil para el trabajo con coordenadas y para el uso posterior de las supercalculadoras pues el cursor tiene esos movimientos. Para ello se puede utilizar tanto el geoplano como el papel cuadriculado, e incluso diseñar actividades lúdicas como la siguiente.

Se juega entre dos. El juego consiste en que el primero le pone una situación al segundo, en una de las dos partes separadas por las ligas (semiplanos y eje de simetría) de modo que el otro lo tenga que hacer igual, pero "al revés", y le añade una nueva situación al primero que debe ponerla igual y ponerle de nuevo una situación al segundo. Y así se va jugando hasta que alguno se equivoque y entonces el otro gana o queden empatados cuando se cumpla el número de jugadas establecidas previamente. 


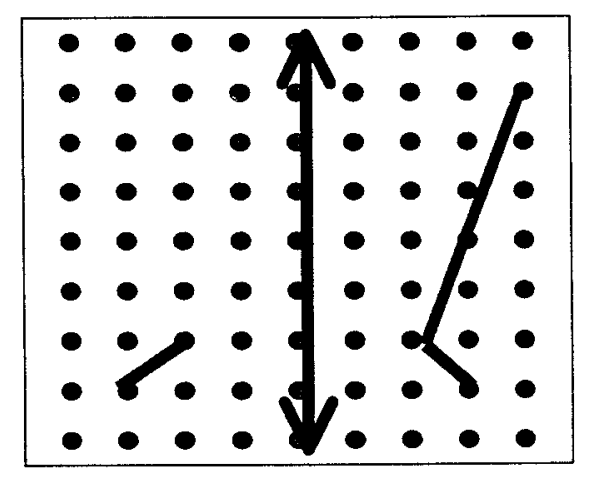

En esta etapa también se tienen que seguir haciendo variaciones de figuras con las nuevas dificultades que se han ido incorporando, explorando propiedades que se conservan o que se pierden.

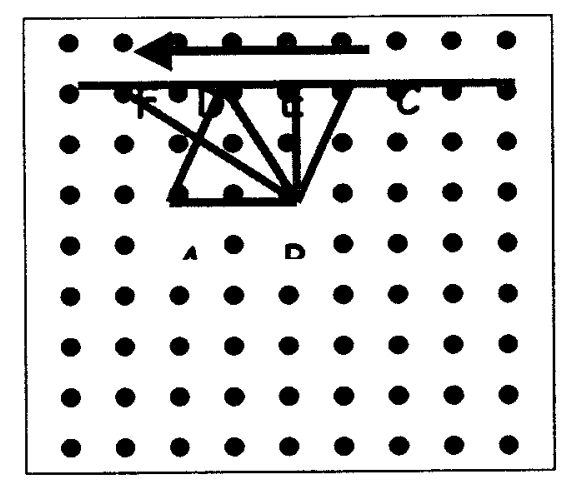

En las exploraciones que se pueden hacer se encuentra, por ejemplo, la de a partir de una figura como el paralelogramo ABCD y dejando solo libre el punto $\mathrm{C}$, moverlo sobre la paralela que pasa por el lado DC. En este caso se conserva el paralelismo de un par de lados pero se pierde el del otro par y la figura deja de ser un paralelogramo para pasar a trapecio (ABED), a triángulo cuando $\mathrm{C}$ y $\mathrm{O}$ coinciden $(\mathrm{ABD})$ y a una figura no convexa que ellos no saben denominar (ABFD). 
En esta etapa también se puede ir preparando el concepto de área y el de perímetro de las figuras geométricas elementales, que dan la posibilidad de ampliar la ejercitación posterior, aprovechando de nuevo el geoplano y la idea de mover una figura.

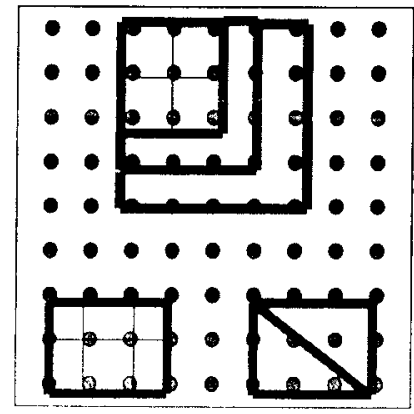

La actividad consiste en determinar en el geoplano figuras elementales, preferentemente empezando por el cuadrado, y mandar a contar los cuadrados que le quedaron dentro. Cualquiera que sea el cuadrado que haya seleccionado cada alumno le va a quedar o $1,4,9 \ldots$ lo que se puede obtener variando la figura original. Es decir: $1,2 \times 2,3 \times 3, \ldots$

Si varían la figura pasándola a un rectángulo, se mantiene la multiplicación del número de cuadrados por cada lado: $3 \times 2$ en este caso.

Se deja planteado el problema de cuál será la cantidad de cuadraditos si lo convierten en un triángulo como el de la figura.

En el tercer momento del desarrollo se está entrando en la etapa denominada racional del aprendizaje de la geometría, por lo que aunque se conservan elementos de la etapa intuitiva operativa, ya se dan pasos en la dirección de un mayor trabajo con los procesos lógicos asociados a razonamientos, básicamente en el planteamiento de conjeturas. 
TERCER MOMENTO DEL DESARROLLO

ETAPA DE EXPLORACIÓN, HACER CONJETURAS Y PRUEBAS.

Inicio de la etapa deductiva igualdad por movimientos geométricos del plano

(simetrías y traslaciones). Mover en una figura. Exploración de propiedades que

se conservan cuando se producen movimientos en una figura. Propiedades básicas

de las figuras elementales. Búsqueda de teoremas relacionados con la

congruencia. Cálculo de perimetros, áreas y volúmenes. Búsqueda de propledades

asociadas a estos conceptos.

Continuación de la ampliación de las ideas sobre la movilidad de las figuras para comprobar experimentalmente, y formalmente, relaciones de congruencia, y conservación de otras propiedades como la igualdad, el paralelismo y la perpendicularidad. Búsqueda de elementos simétricos en una figura y en par de figuras. Continuación del uso del geoplano para ir desarrollando la habilidad de "mover' en una figura. Uso de supercalculadoras (principalmente por el docente) como medio heurístico de apoyo a la exploración, comprensión y búsqueda de casos particulares y límites en la demostración de propiedades. Reproducción (simulación) del alumno usando el geoplano. Uso de la calculadora en la solución de problemas geométricos de cálculo y demostración.

En esta etapa se deben continuar trabajando con actividades donde el alumno tenga que hacer variaciones a figuras con el geoplano, buscar puntos y figuras simétricas en el papel cuadriculado y en el geoplano, similares a las que se vieron en la etapa anterior e incluyendo los casos de figuras propiamente simétricas como el cuadrado, el rectángulo, el triángulo isósceles, el triángulo equilátero, el rombo, la circunferencia, entre otras.

Por ejemplo, determinar las imágenes del círculo negro (más grande) y el rectángulo, por las simetrías de los ejes dados e investigan si el triángulo dado es simétrico. En caso de no serlo, varíarlo para que lo sea y trazar sus ejes de simetría. 


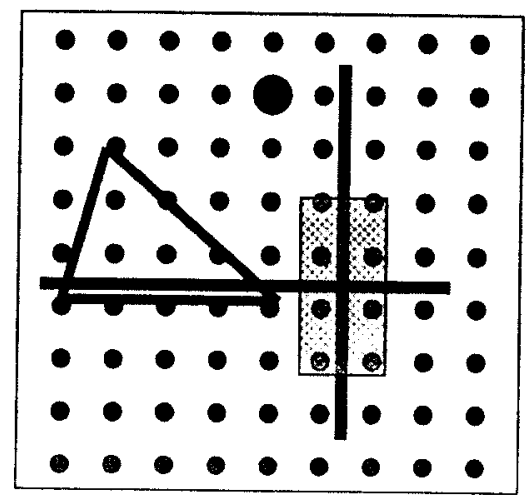

Estamos planteando también en esta etapa la posibilidad de ir combinando el uso del geoplano con la calculadora (para ser utilizada por el alumno en situaciones de problemas) y las supercalculadoras, estas últimas para ser utilizadas como medio de enseñanza y recurso heurístico para el docente. Por ejemplo, ya se le pueden plantear situaciones como la siguiente: ¿Cuál es el rectángulo de mayor área con perímetro de $32 \mathrm{~cm}$ ?

La solución de este problema puede encontrarse tanteando y usando la calculadora, usando el geoplano o usando la supercalculadora. Por ejemplo:

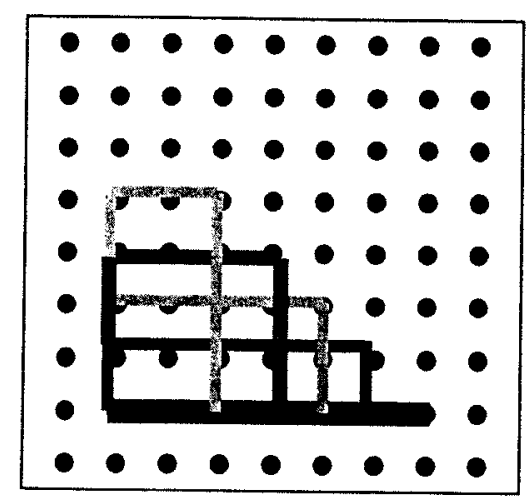


En este caso se ubica en la parte inferior del geoplano la longitud del semiperímetro y se van formando los rectángulos. Se puede ya conjeturar que es el cuadrado, pues el área es de 16 unidades cuadradas. Esto se puede también explorar en la supercalculadora fijando de igual modo un segmento de la longitud del semiperímetro.

Un problema análogo pero de mayor complejidad para su ilustración utilizando recursos tecnológicos es: ¿Cuál es el rectángulo de menor perímetro con un área dada? En este caso el uso del geoplano ya no puede ser por las complejidades que tiene representar una magnitud no lineal como es el área, pero pueden buscarse las ideas tanteando con la calculadora o usando una supercalculadora (se usa como lugar geométrico para mover el punto libremente a una hipérbola del tipo $x y=1)$.

En la última etapa, concebida para el nivel de secundaria (entre 12 y 14 años) ya se puede entrar con más fuerza en la etapa racional y trabajar con más peso la búsqueda de propiedades y sus comprobaciones experimentales o sus demostraciones.

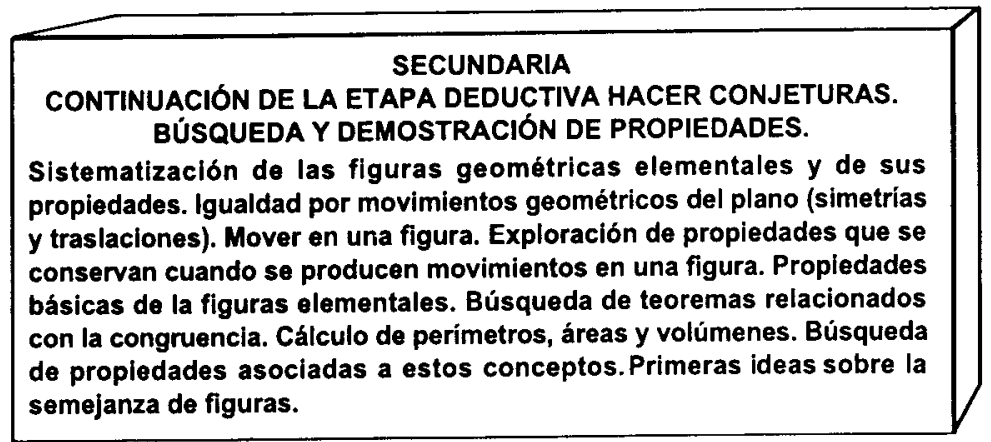


Continuación de la ampliación de las ideas sobre la movilidad de las figuras para comprobar experimentalmente, y formalmente, relaciones de congruencia, y conservación de otras propiedades como la igualdad, el paralelismo y la perpendicularidad. Búsqueda de elementos simétricos en una figura y en par de figuras. Uso de las supercalculadoras por el docente como medio heurístico de apoyo a la exploración, comprensión y búsqueda de casos particulares y límites en la demostración de propiedades y por el alumno para continuar desarrollando la habilidad de "mover" en una figura y también como recurso para la búsqueda de propiedades y de ideas para su demostración, y en la solución de problemas geométricos de demostración, de construcción y de cálculo.

Búsqueda de figuras semejantes y de propiedades asociadas a esta relación.

En esta etapa ya el uso del geoplano puede verse más limitado pues ya hay que eliminar la limitación de la utilización únicamente de cantidades discretas, por ello es mucho más necesario el trabajo con la supercalculadora y para ello iniciar al alumno en el uso de las supercalculadoras. Ello no significa que en ausencia de este medio no se pueda usar el geoplano con las mismas intenciones que en los grados anteriores.

En relación con la organización del contenido en esta etapa, sería deseable que el contenido geométrico clásico que se ha estado trabajando desde los primeros grados, se sistematizara de una manera diferente para propiciar el proceso de búsqueda y evitar que se vuelvan a trabajar de una manera clásica los contenidos. Una manera de organizar este contenido pudiera ser, mediante un enfoque de enseñanza a través de problemas, de la forma siguiente: 


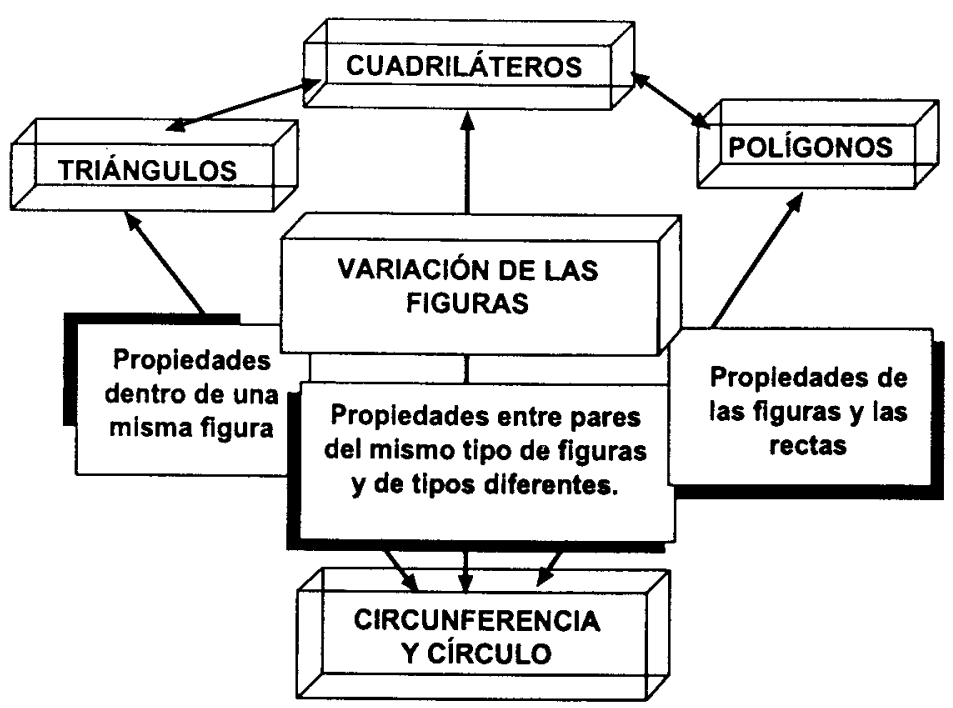

Los problemas que se escojan deben ser problemas abiertos que permitan las búsquedas de los alumnos y la obtención de múltiples propiedades de estas figuras que se irán sistematizando en la medida en que se van obteniendo.

Problemas interesantes pudieran ser:

- Dado un triángulo cualquiera (cuadrilátero o polígono) variarlo de modo de obtener casos especiales y los casos límites. ¿Qué pasaría con la circunferencia?

- Busca condiciones para que dos triángulos puedan superponerse (ser iguales o ser congruentes).

- Busca condiciones para que un triángulo (cuadrilatero, polígono) pueda inscribirse en una circunferencia (circunscribirse). 
Como se puede apreciar es otra manera de concebir la enseñanza aprendizaje de la geometría y de presentar la ejercitación: antes de una manera acabada que no daba posibilidades de exploración, búsqueda, y con este enfoque se tienen todas las potencialidades para ello, de ahí la importancia de poder preparar al alumno desde los grados anteriores para esa flexibilidad en su pensamiento que le permita la exploración, la búsqueda de alternativas y motive en ellos el deseo de investigar y obtener cada vez cosas nuevas para él y sus compañeros.

\section{La Tecnología y la Heurística}

A continuación profundizaremos en la aplicación de las estrategias heurísticas para resolver problemas, como aspecto muy importante de esta nueva concepción en la dirección del proceso de enseñanza aprendizaje de la geometría, y que va dirigido fundamentalmente a los profesores, para facilitarles su trabajo y ayudarlos a generar ideas nuevas es el uso de estas herramientas.

Como antes se planteó, esta concepción permite hacer la introducción de la tecnología, en este caso el empleo de calculadoras, supercalculadoras y computadoras, en el proceso de enseñanza aprendizaje de la geometría, pues con ella existe la posibilidad de "mover las figuras geométricas", es decir, variarlas de modo que adquieran dinamismo, y antes hemos dicho que cuando eso sucede estamos en presencia de la geometría dinámica y esta permite que el alumno se forme una idea más general de esas figuras geométricas y no asocie las propiedades a una forma particular de las figuras, no obstante habría que incluir algunos elementos de contenido, especialmente en lo que a habilidades se refiere, que permitan también que aprendan a "mover en una figura o variarla".

A continuación ilustraremos, con ejemplos desarrollados, 
como se puede utilizar la tecnología para dinamizar la geometría.

\section{Actividad 1. Un estudio sobre el ortocentro}

\section{La situación clásica}

En esta actividad nos referimos a uno de los puntos notables clásicos en los triángulos, el ortocentro. Construyamos un triángulo y tracemos sus tres alturas, lo notable es que las tres se interceptan en un punto. Para convencernos de que no se trata de un fenómeno casual debido a las características del triángulo particular trazado variemos la figura y veremos que se conserva la propiedad.

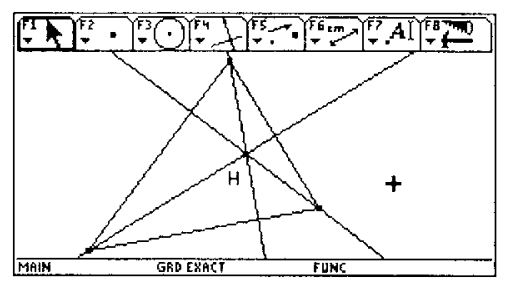

Fig.1

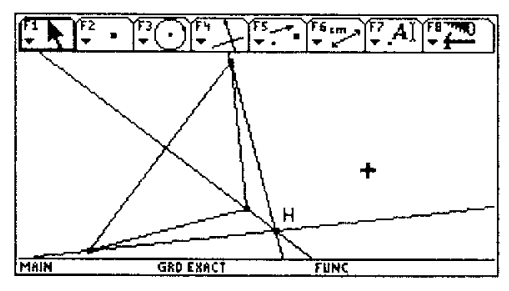

Fig. 2

Las figuras 1 y 2 representan dos casos diferentes, podemos observar que el ortocentro se desplaza del interior al exterior del triángulo al pasar de uno a otro. ¿A qué se debe este desplazamiento? ¿Cómo se mueve el ortocentro al variar el triángulo?

\section{El ambiente de exploración}

En esta primera actividad limitaremos el estudio a un caso especial siguiendo las recomendaciones de la heurística. Consideremos un triángulo inscrito en una circunferencia y estudiemos la variación del ortocentro al variar el triángulo en dicha circunferencia. Para hacer esto necesitamos un triángulo que se mueva conservando la misma circunferencia circunscrita. 
¿Cómo lograr un triángulo que se mueva libremente en una circunferencia?

La construcción debe realizarse de modo que los tres vértices se muevan libremente y para poder observar el movimiento del ortocentro se hace necesario activar la traza para ese punto.

En la figura 3 se ha construido un triángulo con las características dadas y se ha trazado el ortocentro. Par mayor claridad de la figura se han borrado las líneas necesarias para la construcción.

En la figura 4 se recoge la imagen después de realizado el movimiento del vértice $B$ alrededor de la circunferencia y en ella aparece la trayectoria del ortocentro ¿Qué parece ser? ¿Qué propiedades de esa figura se pueden conjeturar?

Repite el proceso con el movimiento de los vértices A y $\mathrm{C}$ respectivamente y observa. ¿Se refuerza o se refuta tu conjetura?

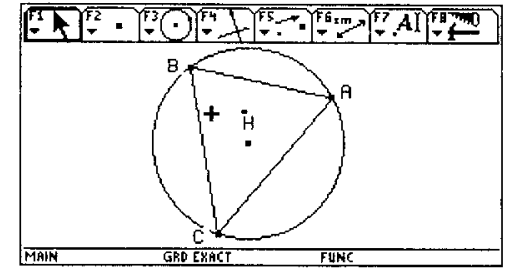

Fig.3

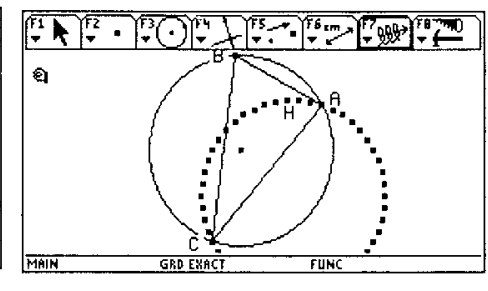

Fig.4

\section{Comprobación de las conjeturas.}

Aparentemente, la trayectoria del ortocentro es una circunferencia congruente con la circunferencia en la que está inscrito el triángulo. En Matemática se hace necesario demostrar o refutar las conjeturas, ¿cómo demostrar éstas? Ana- 
licemos la primera conjetura, se trata de comprobar que la trayectoria es una circunferencia.

¿Qué propiedades conocemos que permitan afirmar que un conjunto de puntos está sobre una circunferencia?

Tenemos en primer lugar los teoremas sobre las condiciones suficientes para que un cuadrilátero sea concíclico, también la caracterización del arco capaz como el lugar geométrico de los puntos desde los cuales se ve un segmento bajo un ángulo fijo. Esta última propiedad parece más prometedora porque está relacionada con la hipótesis de la conjetura.

En efecto el ángulo $\mathrm{B}$ es un ángulo constante inscrito sobre el lado AC, entonces se trata de analizar la relación entre el ángulo formado por las alturas relativas a los vértices $\mathrm{A}$ y C y el ángulo B del triángulo.

Nuevamente la Geometría dinámica nos permite apoyar a los recursos heurísticos. Tracemos las alturas mencionadas y utilicemos el instrumento de medición de la calculadora para obtener el valor de los ángulos implicados.

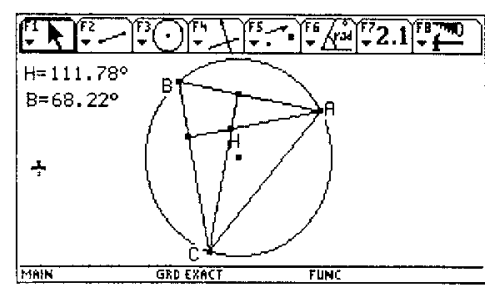

Fig.5

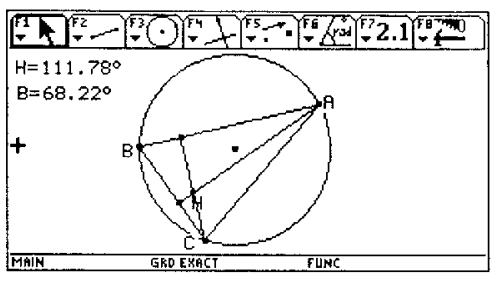

Fig.6

Gracias a la Geometría dinámica podemos ver qué ocurre al variar el triángulo: ambos ángulos permanecen constantes y su suma es $180^{\circ}$, esto sugiere una confirmación de la conjetura y nos apunta una idea para su demostración. En las figuras 5 y 6 se han representado dos posiciones diferentes. 
Para demostrar la conjetura seguimos la idea obtenida anteriormente y nos apoyamos en otra de las estrategias heurísticas: Trazar una figura

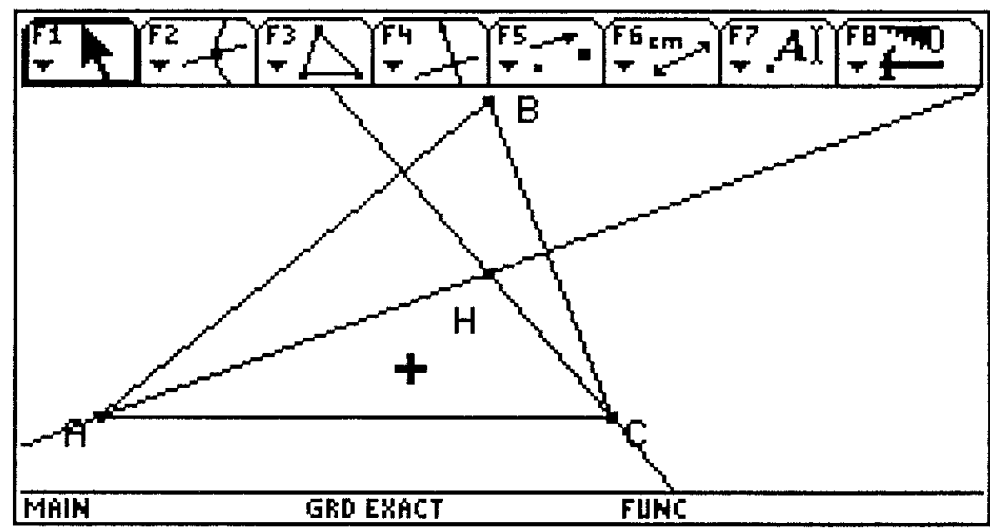

Fig.7

En la figura debemos buscar relaciones entre el ángulo formado por las alturas $(\angle \mathrm{AHC})$ y el ángulo $\mathrm{B}$. Dado que nos interesa que sean suplementarios parece natural investigar la relación con la suma de los ángulos $\mathrm{A}$ y $\mathrm{C}$ (que es suplementaria del ángulo B).

Por ser cada altura perpendicular al ado, obtenemos: $\angle \mathrm{HAC}=90^{\circ}-\angle \mathrm{C}$ y $\angle \mathrm{HCA}=90^{\circ} \angle \mathrm{A}$, luego $\left.\angle \mathrm{HAC}+\angle \mathrm{HCA}=180^{\circ}-\angle \mathrm{A}+\angle \mathrm{C}\right)$ y $\angle \mathrm{AHC}=\angle \mathrm{A}+\angle \mathrm{C}=180^{\circ}-\angle \mathrm{B}$ lo que demuestra nuestra conjetura para el arco más pequeño. De la misma forma podemos comprobarla para el arco mayor.

Nuestra segunda conjetura se refiere a la congruencia de ambas circunferencias. Al buscar resultados referentes a la congruencia de circunferencias, vemos que dos circunferencias son congruentes si y sólo si sus radios son iguales. Esto nos hace pensar en el radio de la circunferencia circunscrita, la relación reconocida es la dada por la ley de los senos: 


$$
\frac{b}{\operatorname{sen} B}=2 R
$$

Dado que en ambos casos se trata del lado AC y los ángulos son suplementarios ambos radios son iguales y las circunferencias son congruentes con lo que queda demostrada nuestra segunda conjetura.

\section{Actividad 2. Rectas y ángulos}

\section{La situación inicial}

En esta actividad nos proponemos estudiar las relaciones que se establecen entre los ángulos determinados al interceptar rectas. La situación más simple se produce al interceptar dos rectas, esta será nuestra situación inicial.

Esta situación se presenta a niños desde los grados iniciales de la escuela y la geometría dinámica permite que estudien en un ambiente de exploración y descubrimiento las relaciones entre las medidas de esos ángulos.

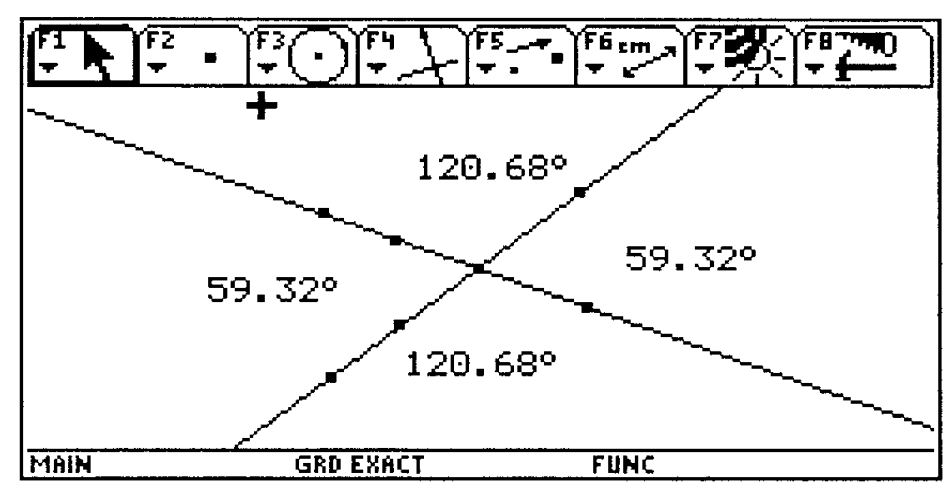

Fig.1 
La figura 1 muestra una situación particular, quedan determinados 4 ángulos y sólo dos medidas, los ángulos son iguales dos a dos.

En esta imagen se puede ver que los ángulos que se oponen por el vértice tienen la misma medida mientras que los que yacen sobre una recta (adyacentes) son suplementarios. Para ver si esto se debe a una posición particular, movemos las rectas y obtenemos que siempre se conserva esta relación como ilustra la figura 2.

Estos resultados son los más elementales de la Geometría y constituyen el punto de partida de la experimentación en esta actividad.

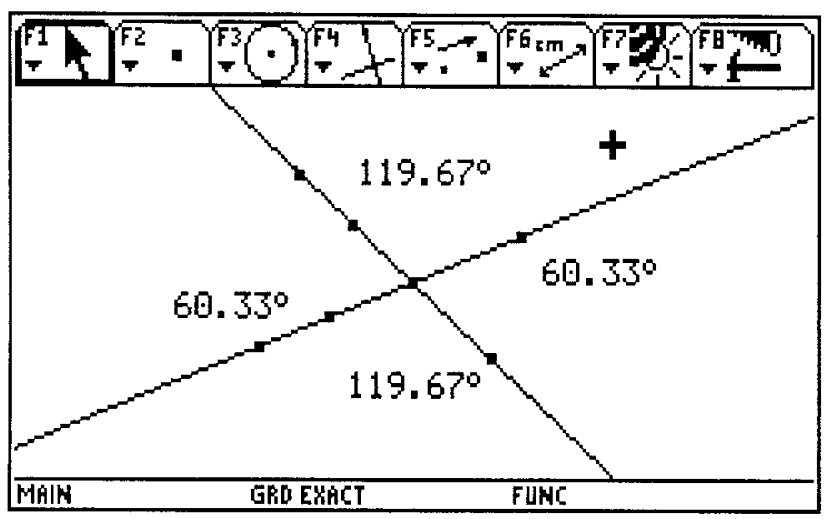

Fig.2

\section{El ambiente de exploración}

La situación que exploraremos, corresponde al caso en el que dos rectas son cortadas por una secante. No asumiremos ninguna posición especial para estas rectas, comenzaremos por considerar dos rectas en posición general y una tercera secante a las dos. 


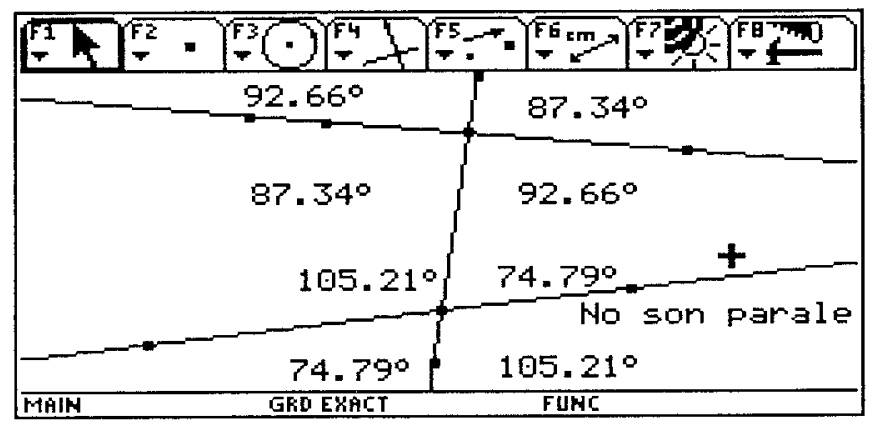

Fig.3

En la figura 3 se representa la situación, el hecho de que las rectas están en posición general se refuerza con el uso de la determinación de propiedades que posee el Cabri. En este caso se puede apreciar que no son paralelas. Al ser dos rectas se tienen 8 ángulos y por la propiedad de que los opuestos tienen igual medida se ven reducidas a cuatro las medidas.

Para la búsqueda de relaciones entre estas medidas, utilizamos la estrategia heurística de mover la figura y comprobamos que en general esta es la situación, se tienen cuatro medidas independientes, se repite la situación analizada al principio pero por duplicado.

Esto se ilustra en la figura 4 en otra posición

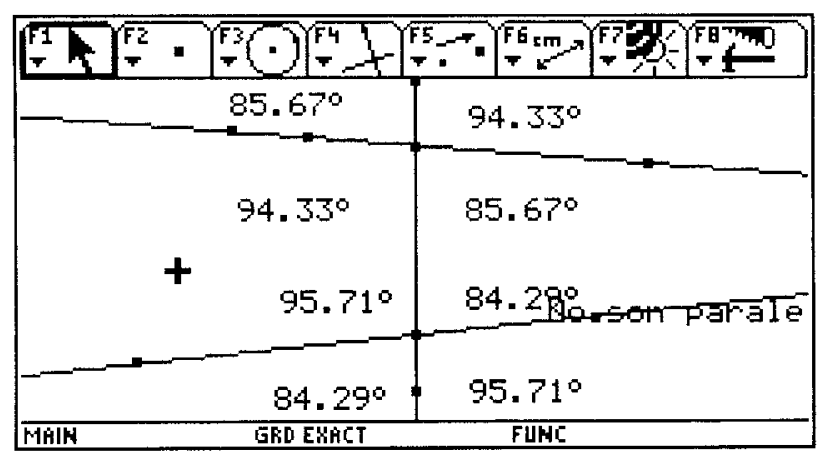

Fig.4 
El uso de los procedimientos heurísticos nos lleva entonces a considerar casos especiales, en esta oportunidad un caso especial se obtiene cuando las rectas son paralelas y se representa en la figura 5 .

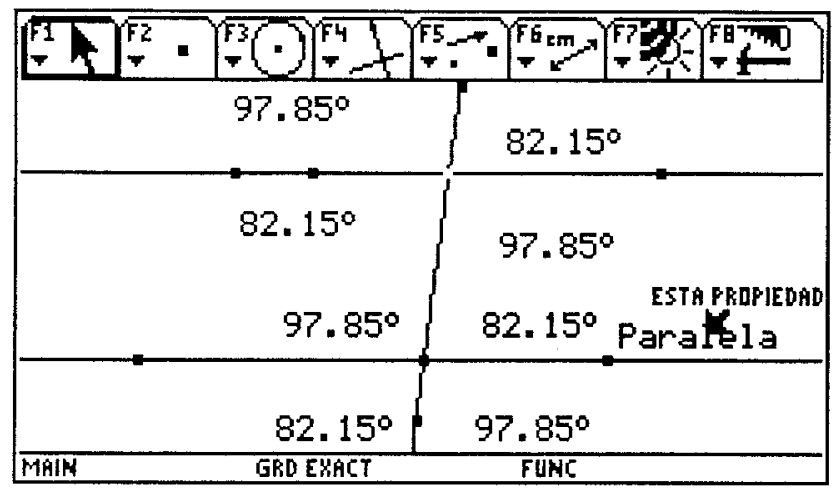

Fig.5

En este caso especial las medidas diferentes se reducen a dos, como en el caso de una sola recta, todos los ángulos agudos tienen la misma medida y todos los ángulos obtusos tienen la misma medida.

Este resultado sintetiza todos los teoremas relativos a ángulos entre paralelas cortadas por una secante y; por otra parte, hace evidente que se trata de un resultado que se ajusta particularmente al caso de las rectas paralelas. Los alumnos pueden comprender que la situación general no conduce a nada nuevo y que se requiere el paralelismo para el nuevo resultado.

\section{Comprobación de las conjeturas.}

Para garantizar la veracidad del resultado, se requiere una demostración. En la búsqueda de la misma también nos apoyamos en la Geometría Dinámica y la heurística. Guiándonos 
por la estrategia de analizar lo dado y lo buscado, vemos en la figura 5 que requerimos comprobar la igualdad de los ángulos que están en la misma posición.

Esa idea ligada al principio de movilidad, nos hace pensar en la posibilidad de desplazar una recta respecto a la otra.

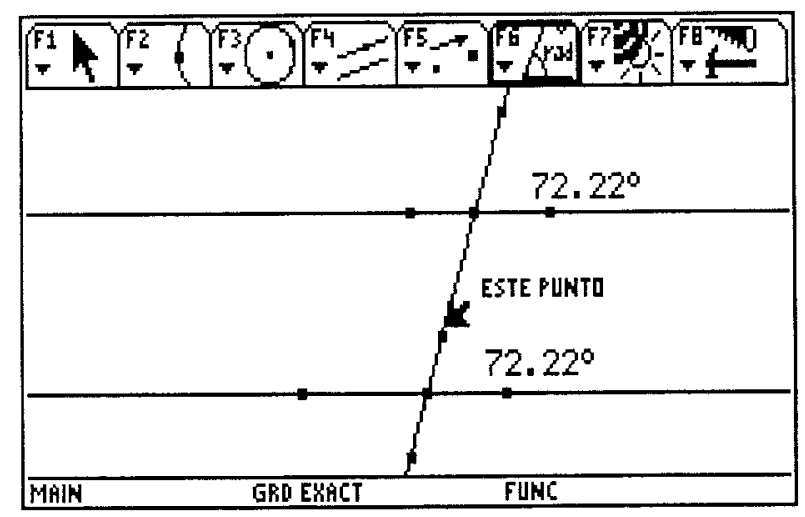

Fig.6

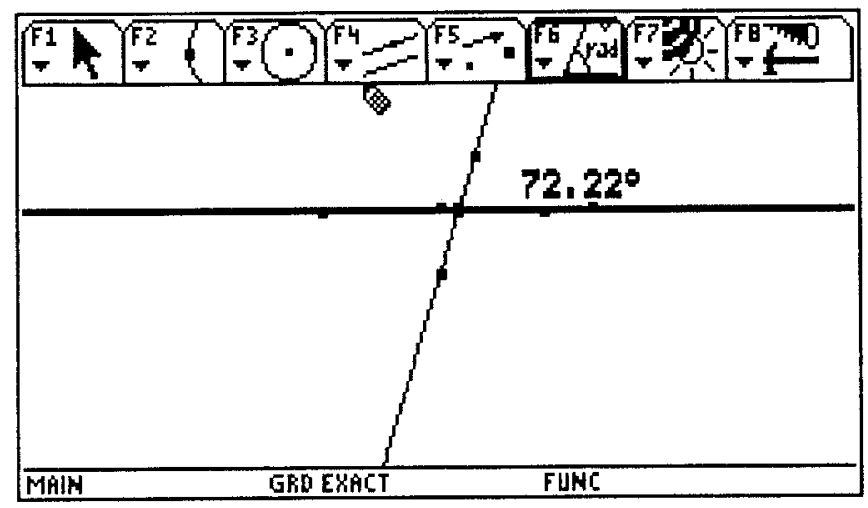

Fig.7

Esta operación se representa en las figuras 6 y 7 , en las que sólo se han representado las medidas de dos ángulos correspondientes para simplificarlas. La figura 7 es el resultado de realizar una traslación en la dirección de la recta secante y se 
puede comprobar que los ángulos de la misma denominación se superponen, es decir, tienen la misma medida.

De esta forma se elabora el siguiente resultado: Cuando dos rectas son cortadas por una secante, si dichas rectas son paralelas, y sólo sí lo son, los ángulos de la misma denominación son iguales y los de diferente denominación suplementarios.

\section{Actividad 3. Un estudio sobre ángulos en un triángulo}

\section{Lo que ya sabemos}

En esta actividad continuaremos investigando sobre ángulos. En la anterior analizamos lo que se puede decir cuando los ángulos se generan al cortarse dos rectas o cuando una recta corta a otras dos $y$, en particular cuando esas dos rectas son paralelas.

En esta actividad nos referimos a tres rectas en posición general que determinan un triángulo y a todos los ángulos que se generan en esa situación.

Dado que se tienen tres puntos en los que se interceptan dos rectas, tenemos en total 12 ángulos que son iguales por pares como se ilustra en la figura 1. .

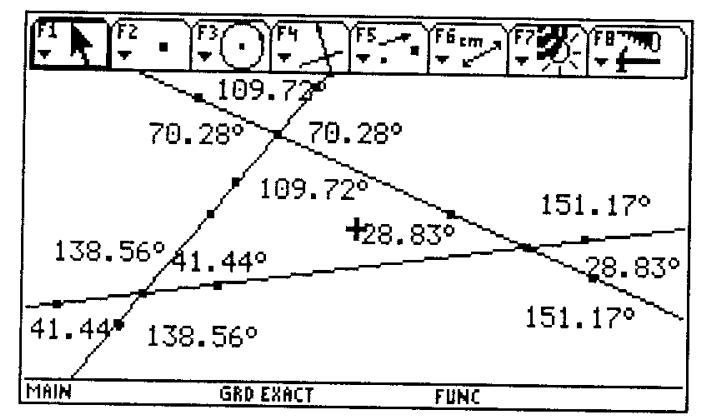

Fig.1 
Resultan entonces seis medidas diferentes que resultan suplementarias dos a dos, con lo que tenemos tres valores independientes como muestra la figura 2 .

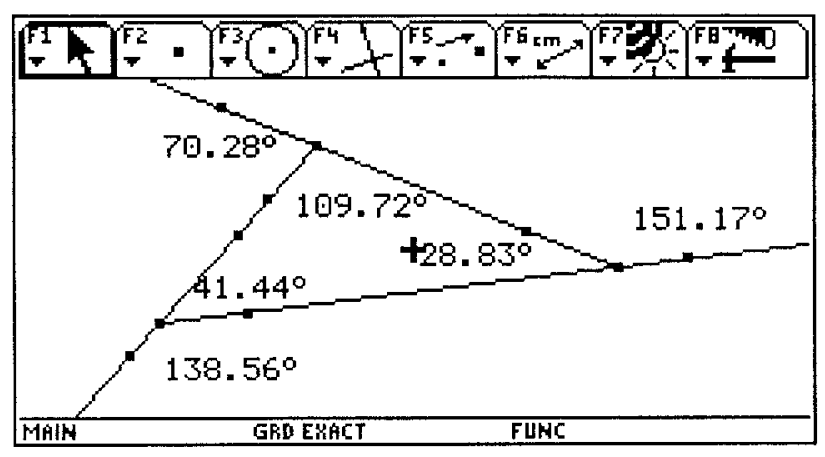

Fig. 2

Nos preguntamos entonces si estos tres valores son realmente independientes o es posible encontrar alguna relación entre ellos. El enfoque clásico consiste en postular directamente la suma de estos valores ya sea que se anuncie de antemano que es $180^{\circ}$ o no. La Geometría dinámica y el uso de la heurística permiten plantear este problema en un ambiente de exploración más libre y abierto a la búsqueda.

\section{El ambiente de exploración}

Ante una situación como la representada en la figura 2 es difícil formular una conjetura razonable, por esta razón recurrimos a la heurística y utilizamos la estrategia de considerar casos particulares. En la situación presente eso significa fijar el valor de alguna de las medidas.

Una idea puede ser considerar que uno de los ángulos es recto, de esa forma quedan sólo dos valores independientes y es más sencillo formular una conjetura. La figura 3 ilustra la situación que resulta. 


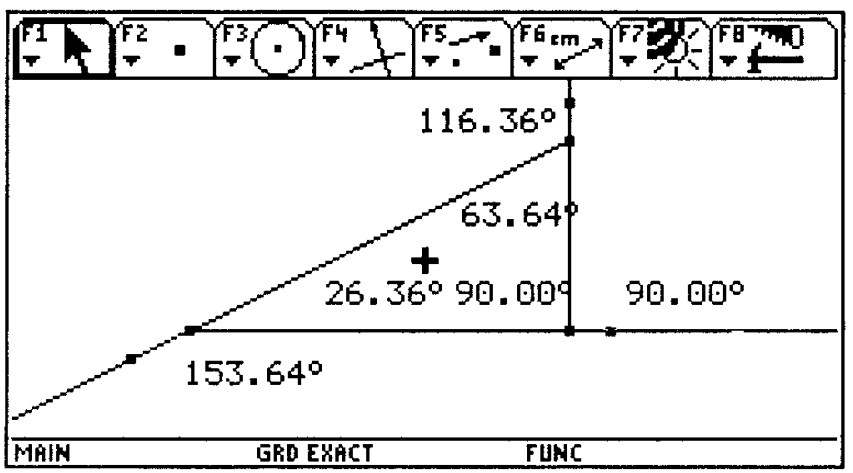

Fig.3

Aquí resultan varias observaciones interesantes:

- La suma de los dos ángulos agudos es igual al ángulo recto.

- La suma de cada uno de los ángulos exteriores es igual a la suma de los dos interiores no adyacentes.

Una de las afirmaciones está muy ligada a la situación particular que hemos elegido, para ver si así se conservan recurrimos a la Geometría dinámica y transformamos la figura. Vemos que ambas observaciones se conservan como ilustra la figura 4.

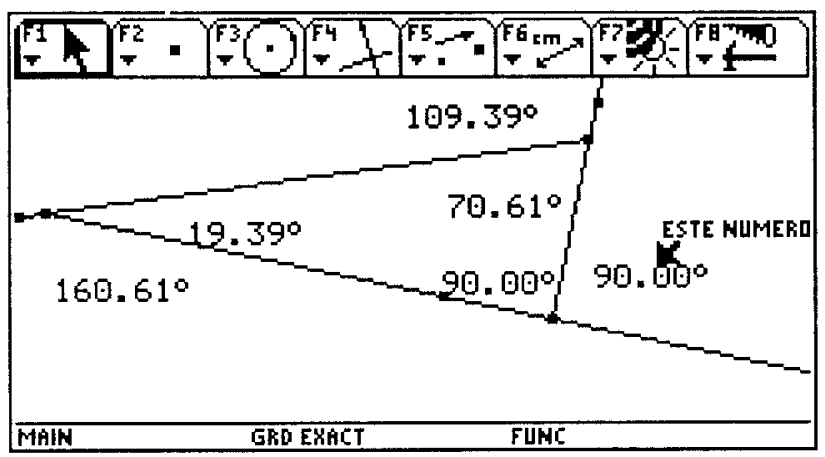

Fig.4 
Ahora es necesario desestimar el caso particular para ver si las afirmaciones se conservan ya que una de ellas no depende de que el triángulo sea rectángulo. Cuando lo hacemos, es decir, prescindimos de la condición de que un ángulo sea recto, vemos que en efecto se conserva la primera afirmación como ilustra la figura 5 .

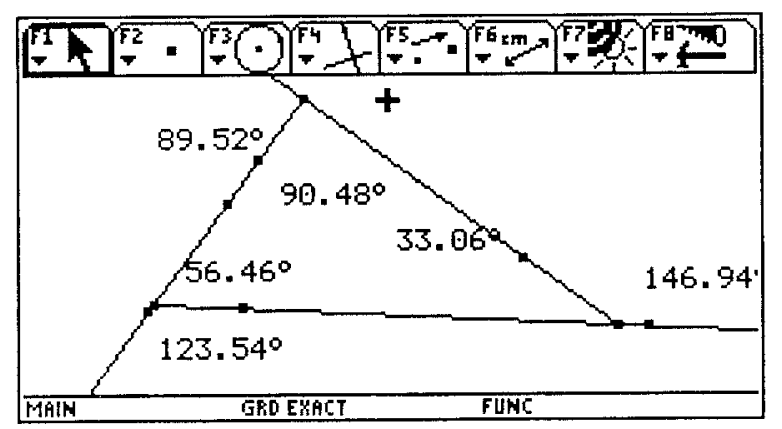

Fig.5

En estas condiciones resulta natural comparar la medida de cada ángulo exterior con la suma de las medidas de los dos interiores no adyacentes pues eso ha sido sugerido por los resultados anteriores.

Con respecto a los ángulos interiores, no se conserva la condición de que la suma de dos de ellos sea igual al tercero, pero podemos interpretar esta relación de una forma más general. En este caso vemos que puede ser interpretado en el sentido de que la suma de los tres es $180^{\circ}$ y esa condición si se ha conservado.

Ahora es natural dar movimiento a la figura y analizar la suma de los ángulos como ilustran las figuras 6 y 7. 


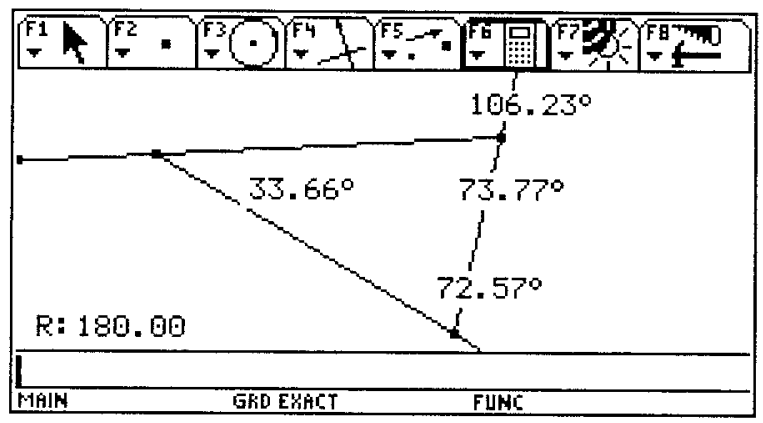

Fig.6

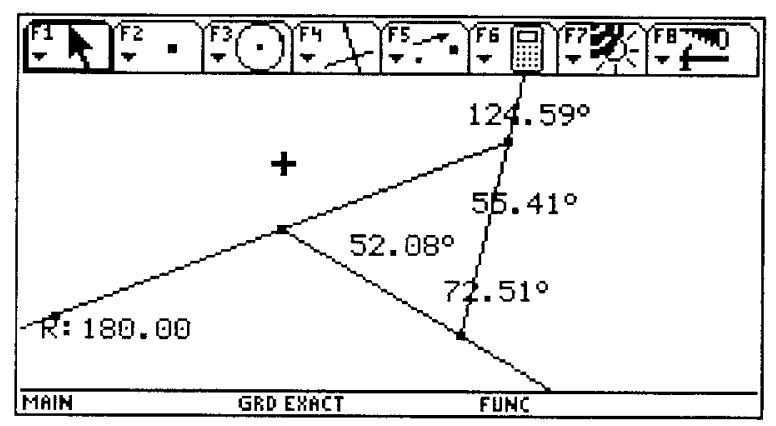

Fig.7

\section{Comprobación de las conjeturas.}

Para la demostración de esta propiedad recurrimos la estrategia heurística de recordar otros resultados relativos a ángulos y de inmediato pensamos en los ángulos entre paralelas. En estas condiciones no hay rectas paralelas pero podemos introducirlas trazando una paralela a uno de los lados.

Eso es lo que hemos hecho en la figura 8 en la que aprovechamos la facilidad de medición de la calculadora para determinar las medidas de los ángulos y comprobar más simplemente el resultado como ilustra la figura 8 . 


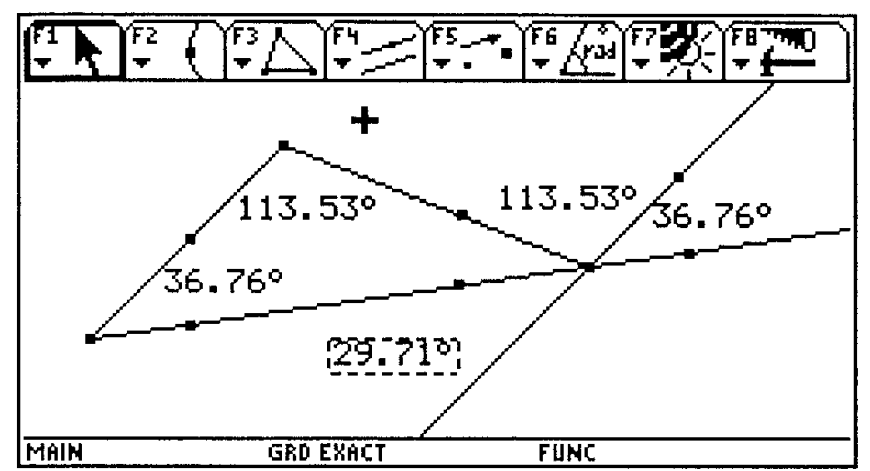

Fig. 8

La recta paralela trazada descompone al ángulo exterior en dos ángulos que son respectivamente iguales a los dos interiores no adyacentes; esa igualdad se justifica porque cada uno de ellos es un ángulo de la misma denominación que uno interior cuando las dos rectas paralelas son cortadas por una secante. En cada caso la secante es la recta que contiene a uno de los lados del triángulo.

A modo de conclusión, los principales cambios en la dirección del proceso de enseñanza aprendizaje están en la utilización de los medios de enseñanza, especialmente el geoplano, calculadoras y supercalculadoras, en la concepción de la ejercitación para darle cabida a este enfoque dinámico y en la utilización de la heurística para propiciar las acciones de búsqueda, lo que implica también un cambio importante en cómo aprender y enseñar la geometría y no tanto en qué contenidos dar.

Los recursos tecnológicos se introducen como un medio que complementa lo previsto en el programa y contribuyen a una mejor comprensión de los contenidos establecidos. 


\section{Bibliografía}

1) Barret, G. y Goebel, J. The impact of graphing calculators in the teaching and learning of Mathematics en teaching and learning Mathematics in the 1990's NCTM Reston 1990

2) Bazán Z. A., Chalini H.A. Estrategias utilizadas por estudiantes egresados de secundaria en la resolución de problemas matemáticos. Revista Especializada de Educación Pedagogía. Tercera Época. Vol. 10. Núm. 6. Invierno 1995. México.

3) Cabañas, Ma. Guadalupe. La técnica de la modelación como un recurso para aprender a resolver problemas. Tesis de Maestría. Universidad Autónoma de Guerrero. México. Julio de 1995.

4) Campistrous, L. y Celia Rizo. Aprende a resolver problemas aritméticos. Editorial Pueblo y Educación. Ciudad de la Habana. 1997.

5) Campistrous, L. y Rizo, Celia(1999). Algunas técnicas de resolución de problemas aritméticos. Publicaciones del Congreso Pedagogía 99. Habana 1999.

6) Campistrous, L. y Rizo, Celia (1996). Aprende a resolver problema aritméticos. Editorial Pueblo y Educación. Habana.

7) Campistrous L y Jorge López (2001). La calculadora como una herramienta heurística. Revista Uno. Razonamientos y Pruebas. No. 28. Editorial Grao. Barcelona España.

8) Cedillo Avalos, Tenoch (1998). La calculadora en el salón de clase. Sentido Numérico e Iniciación al Algebra. Grupo Editorial Iberoamérica. México.

9) Cedillo, Tenoch y Santillán, M. De la aritmética al álgebra: una experiencia para desarrollar habilidades de representación simbólica empleando como recurso tecnológico calculadoras y computadoras en Memorias de la 48 Reunión Centroamericana Y del Caribe sobre formación de profesores e investigación en Matemática Educativa pp.223-228 México DF 1990.

10) Cervera, Pablo Algunas estrategias para la resolución de problemas geométricos en $12^{\circ}$ grado. Tesis de Maestría en Educación Superior Santiago de Cuba 1999. 
11) Cordero, F. Y Solís, M. Las gráficas de las funciones como una argumentación del cálculo Grupo Editorial Iberoamérica, México D.F. 1995

12) DF 1990

13) Hewhkowitz, Rina y otros. El papel de la Geometría en la formación general Actas del $7^{\circ}$ Congreso internacional sobre Educación Matemática pp.160-227 U. Lava1 1994

14) Hitt Espinosa, Fernando, 1994. "Educación Matemática y uso de nuevas tecnologías". Perspectivas en Educación Matemática. Ernesto Sánchez y Manuel Santos (Editores). Departamento de Matemática Educativa del Centro de Investigación y Estudios Avanzados el IPN. México. Páginas 21 a 42.

15) Johnson, D.A. Un modelo para la investigación en la clase de matemáticas. Revista "El maestro de matemáticas". No. 59. USA. 1966.

16) Lezcano, Luis E. Algunas reflexiones sobre las estrategias de resolución de problemas en Publicaciones de la 98 Reunión Centroamericana y del Caribe sobre formación de profesores e investigación en Matemática Educativa pp.401-406 Habana 1995.

17) López, Jorge M. y Luis Campistrous. (2001) La calculadora como herramienta heurística. Revista Uno. No. 28. Editorial Grao. Barcelona.

18) López, Jorge M. (2000) Actividades matemáticas para la calculadora TI85 Universidad de Puerto Rico. Rio Piedras.

19) López, Jorge M. (2000). La calculadora simbólica en la enseñanza del cálculo Artículo inédito Universidad de Puerto Rico. Río Piedras.

20) Martínez Cruz, Armando Gráficas como medio y no como objetivo: el impacto de las calculadoras gráficas en cálculo y precálculo en Publicaciones de la 98 Reunión Centroamericana y del Caribe sobre formación de profesores e investigación en Matemática Educativa pp.119-124 Habana 1995.

21) Mariñez; Armando Explorando transformaciones de funciones con una calculadora gráfica en Memorias de la X Reunión Centroamericana $\mathrm{Y}$ del Caribe sobre formación de profesores e investigación en Matemática Educativa 557-561 Puerto Rico 1996. 
22) Martínez, P. y Block S., D. La calculadora como apoyo para la nivelación en aritmética en la escuela primaria. en Memorias de la X Reunión Centroamericana y del Caribe sobre formación de profesores e investigación en Matemática Educativa 602-608 Puerto Rico 1996. 